\title{
Integrated Modeling and Experimental Programs to Predict Brine and Gas Flow at the Waste Isolation Pilot Plant
}

\author{
R.L. Beauheim, S.M. Howarth, P. Vaughn, S.W. Webb, and K.W. Larson \\ Sandia National Laboratories
}

(USA)

\begin{abstract}
Evaluation of the performance of the WIPP repository involves modeling of brine and gas flow in the host rocks of the Salado Formation, which consist of halite and higher permeability anhydrite interbeds. Numerous physical, chemical, and structural processes, some of them coupled, must be understood to perform this modeling. Gas generation within the repository, for example, is strongly coupled to the amount of brine inflow to the repository because brine aids in the corrosion of metals and associated generation of hydrogen gas. Increasing gas pressure in the repository, in turn, decreases the rate of brine inflow. Ultimately, the gas pressure may exceed the brine pressure and gas may flow out of the repository. The initial models used by WIPP Performance Assessment (PA) were simplified because of a lack of WiPP-specific data on important processes and parameters. Relative-permeability curves and a correlation between threshold pressure and permeability taken from studies reported in the literature were used in PA models prior to being experimentally verified as appropriate for WIPP. In addition, interbed permeabilities were treated as constant and independent of effective stress in early models. Subsequently, the process of interbed fracturing (or fracture dilation) was recognized to limit gas pressures in the repository to values below lithostatic, and assumed (and unverified) relationships between porosity, permeability, and pore pressure were employed. Parametersensitivity studies performed using the simplified models identified important parameters for which sitespecific data were needed. Unrealistic modeling results, such as room pressures substantially above lithostatic, showed the need to include additional processes in the models. Field and laboratory experimental programs have been initiated in conjunction with continued model development to provide information on important processes and parameters. Current field experiments are aimed at determining the permeability of anhydrite and halite beds under undisturbed conditions, the pressuredependence of anhydrite fracture permeability, and the threshold pressure of anhydrite fractures. Laboratory experiments are being performed on anhydrite core samples to determine capillary-pressure curves, relative-permeability curves, and permeability and porosity as a function of applied stress. The current PA models built upon these experimental data are considerably more realistic and credible than the initial models used and, in some cases, predict significantly different consequences.
\end{abstract}

\section{DISCLAIMER}

This report was prepared as an account of work sponsored by an agency of the United States Government. Neither the United States Government nor any agency thereof, nor any of their employees, makes any warranty, express or implied, or assumes any legal liability or responsibility for the accuracy, completeness, or usefulness of any information, apparatus, product, or process disclosed, or represents that its use would not infringe privately owned rights. Reference herein to any specific commercial product, process, or service by trade name, trademark, manufacturer, or otherwise does not necessarily constitute or imply its endorsement, recommendation, or favoring by the United States Government or any agency thereof. The views and opinions of authors expressed herein do not necessarily state or reflect those of the United States Government or any agency thereof. 


\section{DISCLAIMER}

Portions of this document may be illegible in electronic image products. Images are produced from the best available original document. 


\section{Introduction}

The Waste Isolation Pilot Plant (WIPP) is a repository for transuranic wastes located $655 \mathrm{~m}$ below ground surface in bedded evaporites of the Permian-age Salado Formation. The WIPP site is located near Carlsbad, New Mexico, USA (Figure 1). One scenario of concern to the performance of WIPP is the generation and migration of gas from the repository. While gases are not themselves of direct regulatory concern, they may serve to mobilize (facilitate the transport of) volatile organic compounds (VOC's) present in the waste. Transport of VOC's across the WIPP site boundary is subject to regulation.

The gas generation and migration scenario involves a number of interrelated processes. Among these is brine inflow caused by pressure disequilibrium between the repository, initially at atmospheric pressure, and the fluid in the surrounding rock, which because of the plasticity of halite, is at a pressure approaching lithostatic. The majority of the brine entering the repository comes not through the halite layers that make up most of the stratigraphy, but through thin anhydrite interbeds containing naturally occurring fractures. The brine entering the repository reacts with metals present in the waste and drums to form hydrogen gas. Other gases are generated by

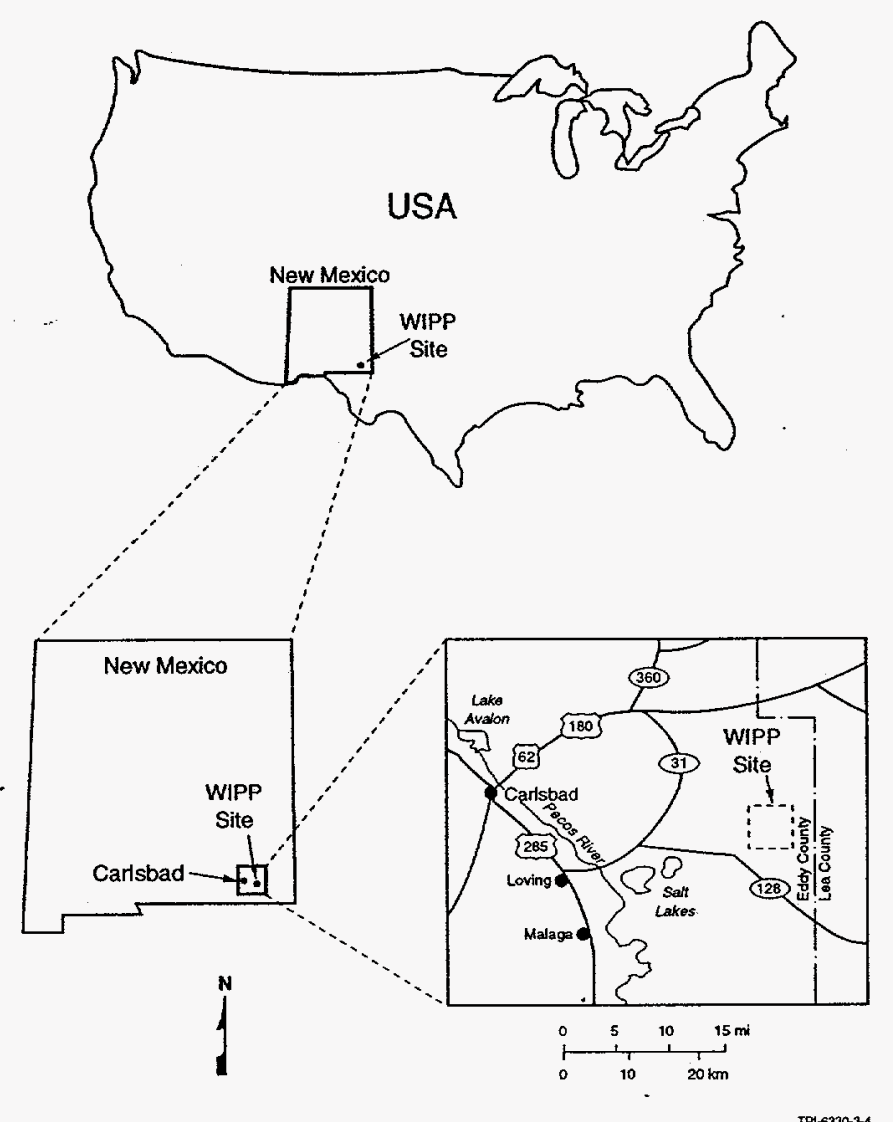

Figure 1. Location of the WIPP site. microbial degradation of organic materials present in the waste. As gases accumulate and the surrounding salt creeps inward, the pressure within the repository rises. Eventually the gas pressure in the repository exceeds the brine pressure in the surrounding rock. At this point there are two limits on the ability of the gas to leave the repository. First, the rock may have a threshold pressure, which is the pressure increment above the brine pressure that gas must achieve before it can begin to displace brine from the pore spaces in the rock and establish continuous flow paths. Second, the permeability of the rock, either intrinsic or relative, may be too low to allow the gas to flow away as fast as it is being generated. If this occurs, the pressure in the repository may continue to rise.

Just as they provide the primary pathway for brine to enter the repository, so will the anhydrite interbeds provide the most likely pathway for gas to leave the repository. Because of their higher permeabilities relative to halite, the interbeds have lower threshold pressures and admit gas more readily than halite. Once gas enters the interbed fractures, the two-phase flow characteristics (capillary pressure and relative permeability) of the rock will control the rate at which the gas can flow away. If this rate is lower than the rate at which gas is being generated, the pressure in the interbed fractures 
will rise. As this occurs, the fractures may dilate, increasing both in permeability and storage capacity. This dilation may continue until the enhanced permeability and storage can accommodate the gas being generated without additional increase in pressure.

Modeling of the gas generation and migration scenario requires knowledge (or assumptions) of the parameters and processes involved. The intrinsic permeability and far-field pore pressure in the anhydrite interbeds have been characterized by an in situ testing program (Beauheim et al., 1991, 1993). Information on two-phase flow properties, threshold pressure, and pressure-dependent permeability (fracture dilation) is less easily obtained. The strategy employed at WIPP for setting experimental priorities is to first make assumptions about properties and processes that bound the expected ranges, and then perform model calculations to determine if the uncertainties affect evaluation of repository performance. If uncertainty in a particular area can affect whether or not calculated performance complies with regulations, an experimental program to reduce the uncertainty may be warranted. In some cases, an iterative sequence of initial models, initial experiments, new models, and new experiments is required to resolve areas of uncertainty adequately. This paper describes how three modeling and experimental programs have been integrated to address the WIPP gas generation and migration scenario.

\section{Two-Phase Flow Properties}

Characteristic curves for capillary pressure and relative permeability are used to determine the pressures and rates at which gas is able to leave the repository and enter anhydrite interbeds. The capillary-pressure function (Figure

2a) describes the pressure at which gas can first enter a brinesaturated medium (defined here as the threshold pressure) and the pressure disequilibrium that occurs between brine and gas as the gas saturation of the medium increases. The relativepermeability function (Figure $2 b$ ) describes how the relative permeabilities of the medium to brine and gas change as gas saturation increases and brine saturation decreases. Both capillary pressure and relative permeability must be measured at known saturations to define the characteristic curves. No method is known to determine saturations under field, or in situ, conditions. Characteristic curves, therefore, must be defined from tests on core samples in a laboratory where saturations can be controlled and measured.

Before undertaking these measurements, modeling studies were performed using different sets of characteristic curves with

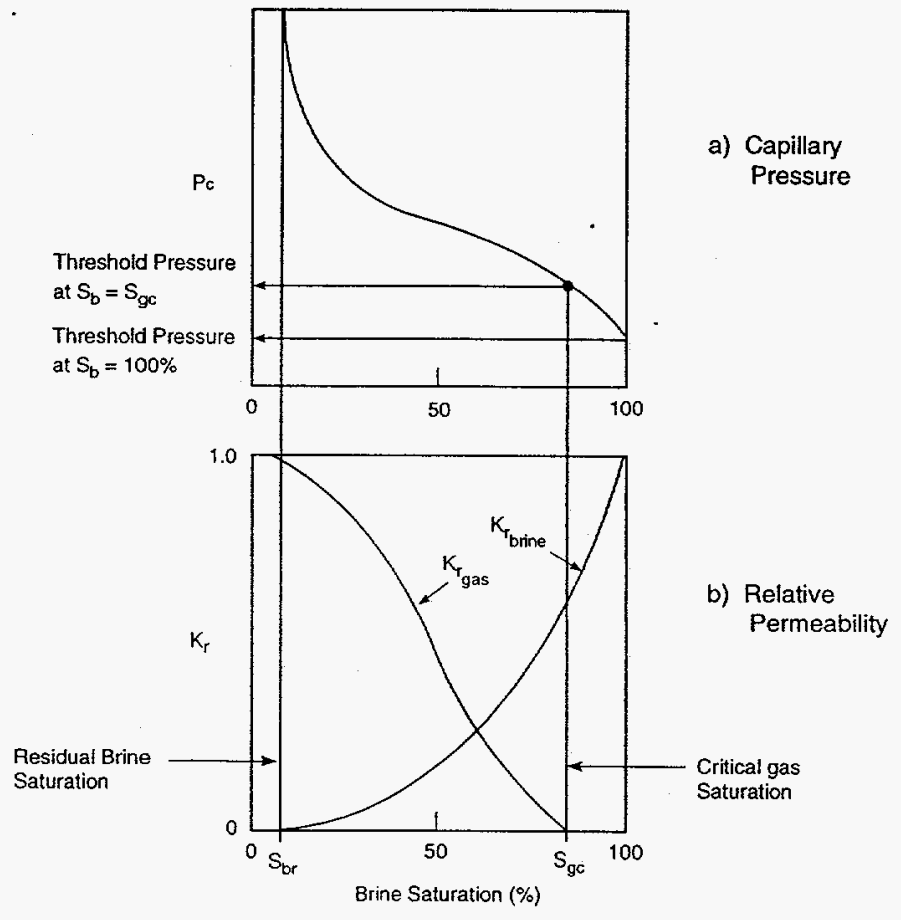

TA1-615.69.0

Figure 2. Relationship between (a) capillary pressure and (b) relative permeability. 
widely different forms. Webb (1992a) studied the effect of the Brooks and Corey (1964) curves and the Sandia Functions (Pruess, 1987), which are based on van Genuchten (1980). The two-phase characteristic curves can have a dramatic effect on the gas-migration distance. Subsequently, similar studies were performed using the Brooks and Corey and van Genuchten/Parker curves (WIPP Performance Assessment Department, 1992). The van Genuchten/Parker curves are similar to the Sandia Functions except the nonwetting phase relative permeability is given by Parker et al. (1987). Again, the gas-migration distance was found to be sensitive to the two-phase characteristic curves.

The forms of the two-phase curves employed are shown in Figures 3 and 4 . As shown in Figure 3, the Brooks and Corey and van Genuchten capillary-pressure curves have a similar shape except approaching full liquid saturation. The Brooks and Corey curve (Figure 3a) has a threshold pressure on the order of $1 \mathrm{MPa}$ for WIPP materials, while the van Genuchten relationship (Figure 3b) has zero threshold pressure. Figure 4 presents the relativepermeability curves. The wetting-phase curves for both models are similar, although the Brooks and Corey value is always higher than that of van Genuchten. The nonwetting-phase (gas) relationships are dramatically different. For Brooks and Corey (Figure $4 a)$, the gas relative permeability is zero until a critical gas saturation $(20 \%)$ is reached. The relative permeability slowly increases with increasing gas saturation. In contrast, the Parker nonwetting-phase relative permeability (Figure 4b) does not have a critical gas saturation, so any amount of gas is mobile. In addition, the Parker relative-permeability curve increases steeply with increasing gas saturation and at any saturation is considerably higher than the Brooks and Corey curve. Webb (1992b) discusses the effect of characteristic curves on gas-saturation profiles, including both Brooks and Corey and van Genuchten/Parker curves. Both the Brooks and
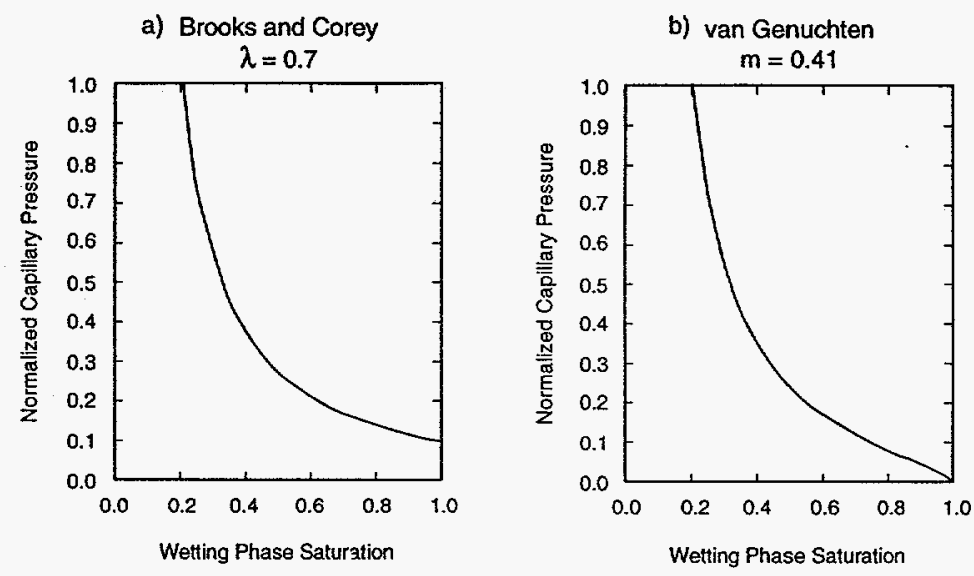

Figure 3. Example capillary-pressure curves used by WIPP PA.
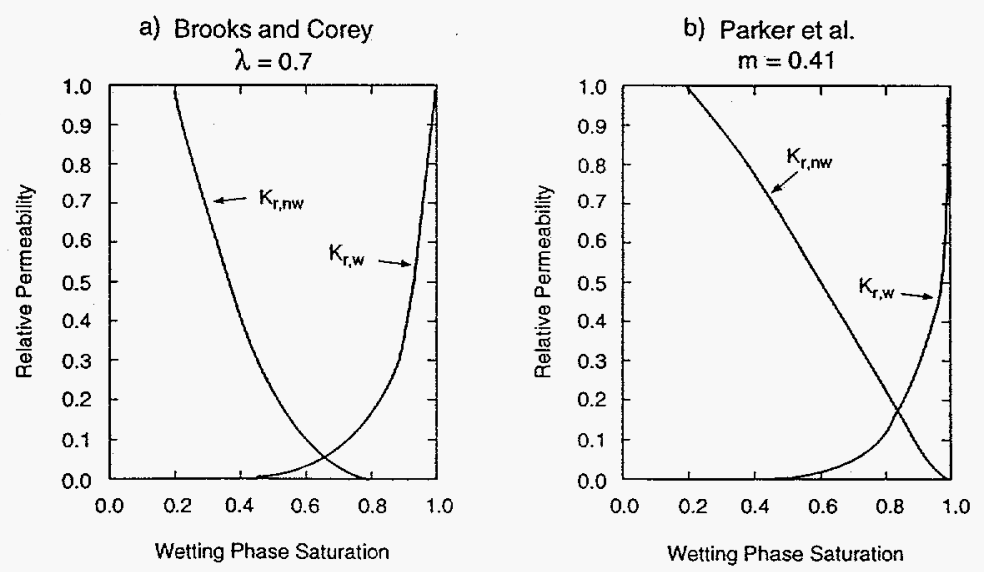

Figure 4. Example relative-permeability curves used by WIPP PA. 
Corey and van Genuchten/Parker curves used by WIPP PA were developed from studies of porous media. Their applicability to fractured media is, therefore, uncertain, but no characteristic curves developed specifically for fractured media are known.

Modeling of gas migration from the repository was performed assuming both flat-lying (horizontal) stratigraphy and a $1^{\circ}$ dip more representative of actual conditions at the WIPP. The modeled stratigraphy included single anhydrite interbeds above and below the repository horizon. In all simulations, gas-migration distances were greater in the upper interbed than in the lower interbed. The modeling shows that the selection of Brooks and Corey or van Genuchten/Parker characteristic curves has a large effect on the calculated gas-migration distance. The Brooks and Corey curves, because they can include a threshold pressure greater than zero, a critical gas saturation of $20 \%$, and low relative permeability to gas at low gas saturations, tend to restrict gas migration, keeping it within about $1 \mathrm{~km}$ of the repository in the upper interbed over a 10,000-yr period in the simulation shown in Figure 5a. Use of van Genuchten/Parker curves in an otherwise identical simulation causes gas to migrate over $5 \mathrm{~km}$ from the repository in the upper interbed over 10,000 $\mathrm{yr}$ (Figure $5 \mathrm{~b}$ ). Gas saturations within the interbeds, however, are much lower using the van Genuchten/Parker curves because those curves include neither threshold pressure nor a critical gas saturation. The effect of $1^{\circ}$ dip on the simulations is shown in Figures $6 \mathrm{a}$ and $6 \mathrm{~b}$. Using Brooks and Corey curves, gas has a slight tendency to move preferentially up dip, but is still confined within a radius of about $1 \mathrm{~km}$ over $10,000 \mathrm{yr}$.
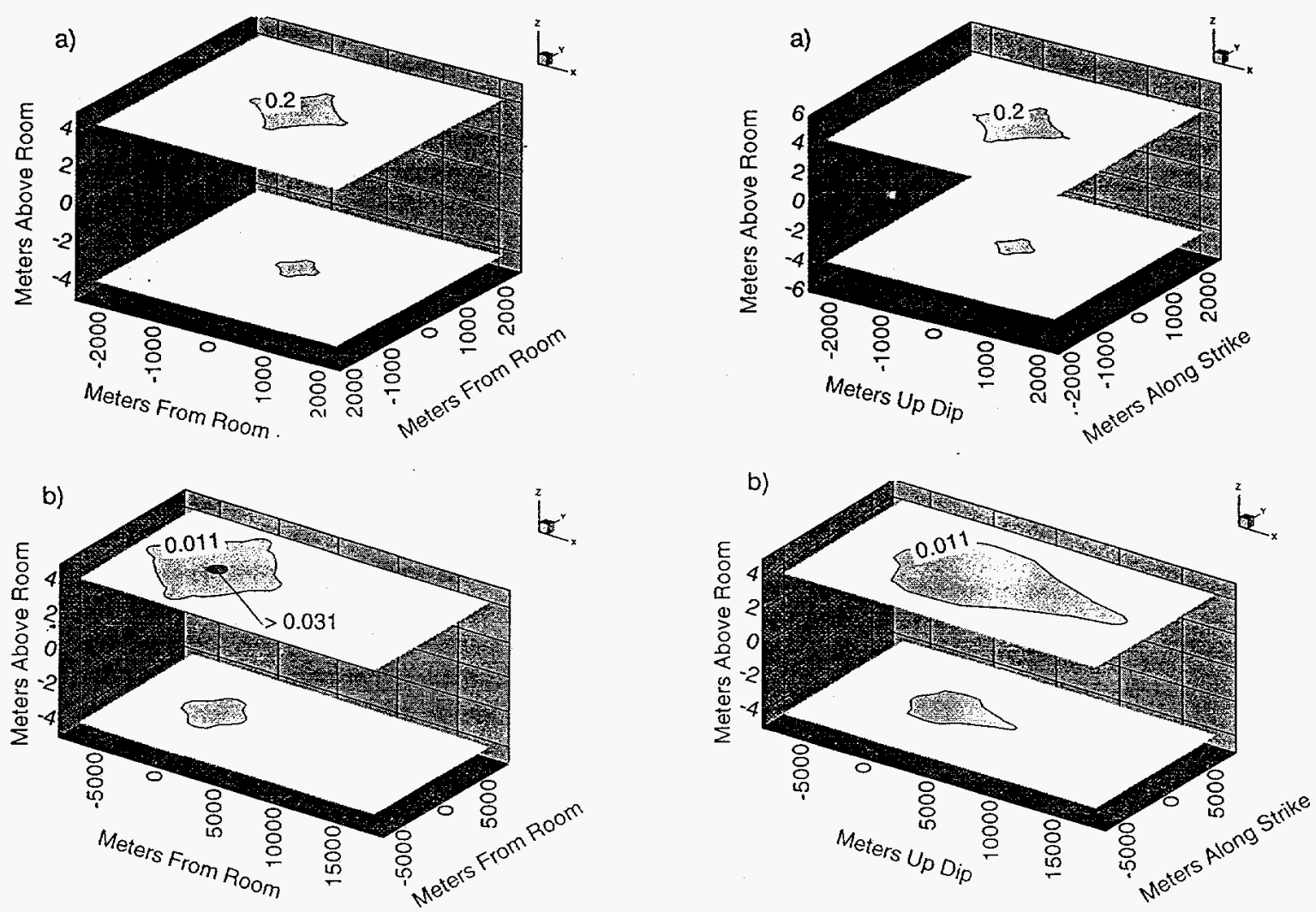

Figure 5. Gas saturations at 10,000 years in horizontal anhydrite interbeds using (a) Brooks and Corey characteristic curves and (b) van Genuchten/Parker characteristic curves.

Figure 6. Gas saturations at 10,000 years in anhydrite interbeds with $1^{\circ}$ dip using (a) Brooks and Corey characteristic curves and (b) van Genuchten/Parker characteristic curves. 
Preferential up-dip movement is much more pronounced using van Genuchten/Parker curves (Figure $6 \mathrm{~b})$. The simulated gas-migration distance in the upper interbed increases to about $15 \mathrm{~km}$ over $10,000 \mathrm{yr}$, relative to the horizontal simulation (Figure $5 \mathrm{~b}$ ).

These simulations showed that knowledge of the true nature of the characteristic curves for anhydrite interbeds is needed to perform realistic modeling of gas migration. A laboratory test program is currently underway to provide the needed two-phase flow characteristics. Initial experiments included determination of capillary-pressure curves for 12 core samples under no confining pressure using centrifuge and mercury-injection techniques (Figure 7). The capillary-pressure results from the oil-air centrifuge tests and the mercury-air mercury-injection tests were corrected for air-brine conditions for WIPP applications. These data could be fit with either a van Genuchten/Parker model or a Brooks and Corey model with a low threshold pressure. Additional measurements are planned in 1995 of capillary pressure and relative permeability under a range of confining pressures to provide characteristic curves that may be used directly in future simulations of gas migration. Reliable extrapolation of data from the laboratory scale to the repository scale is always of concern because of uncertainty about whether or not the features or conditions controlling two-phase flow on the repository scale are, or can be, adequately characterized in small core samples. No alternative to this extrapolation exists, however, because saturations cannot be controlled and measured on the repository scale.

\section{Gas Threshold Pressure}

The gas threshold pressure, in the usage adopted for this paper, is the pressure at which gas establishes an interconnected flow path through a porous (or fractured) medium. It corresponds to

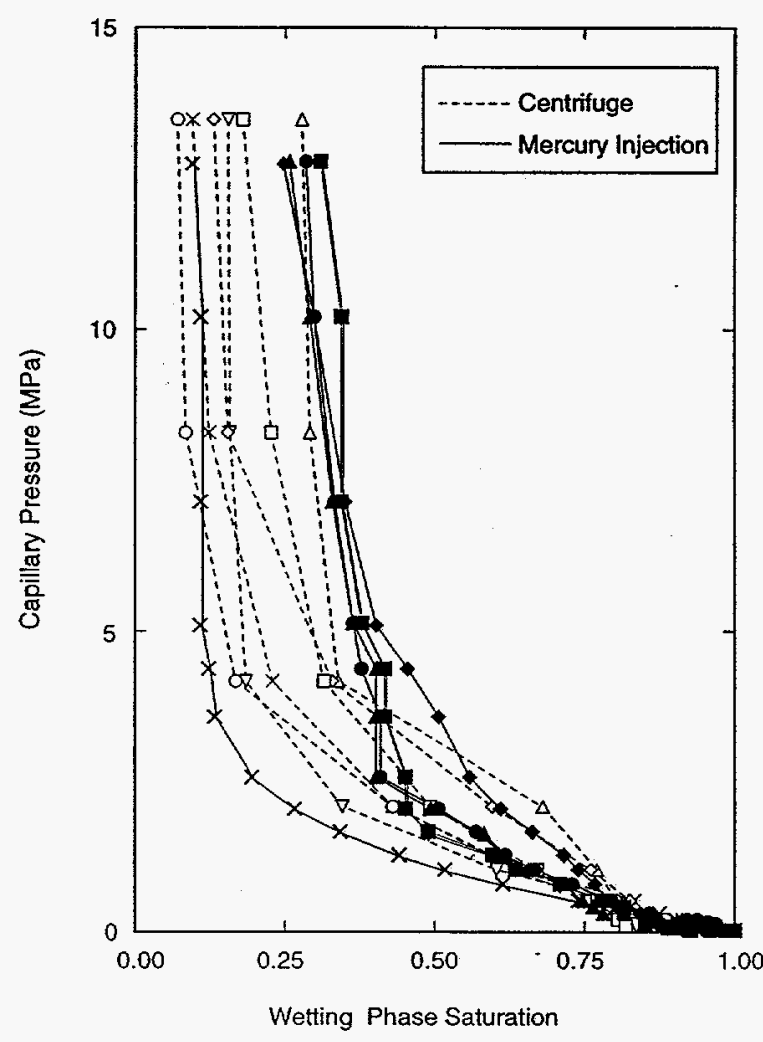

Figure 7. Laboratory capillary-pressure data from tests on anhydrite core. the point on a capillary-pressure curve at the critical gas saturation. Davies (1991) compiled literature data available on threshold pressure and found that threshold pressure generally increases as intrinsic permeability decreases (Figure 8 ). He defined correlations between intrinsic permeability and threshold pressure for a variety of lithologies, including anhydrite. Given the range of vertically averaged permeabilities obtained from in situ testing of anhydrite interbeds at WIPP $\left(6 \times 10^{-20}\right.$ to $\left.1 \times 10^{-18} \mathrm{~m}^{2}\right)$, Davies' correlation for anhydrite indicated that the gas threshold pressure of these beds might lie between 0.1 and $5 \mathrm{MPa}$. If threshold pressures truly were as high as $5 \mathrm{MPa}$, then hydraulic (or pneumatic) fracturing of the host rock surrounding the WIPP repository might occur before gas would enter the rock.

Laboratory threshold-pressure experiments were performed to determine if the threshold pressures of Salado anhydrites were really so high as to make hydraulic fracturing a possibility. Threshold pressures ranging from 0.016 to $0.003 \mathrm{MPa}$ were determined from six mercury-injection tests 
performed on anhydrite core samples having permeabilities between $4 \times 10^{-19}$ and $4 \times 10^{-18}$ $\mathrm{m}^{2}$. These threshold pressure values are one to two orders of magnitude lower than predicted by Davies' correlation. The tests, however, were conducted under no confining pressure, which may have contributed to the low values. Additional laboratory tests are planned under a range of confining pressures to provide results more representative of in situ conditions.

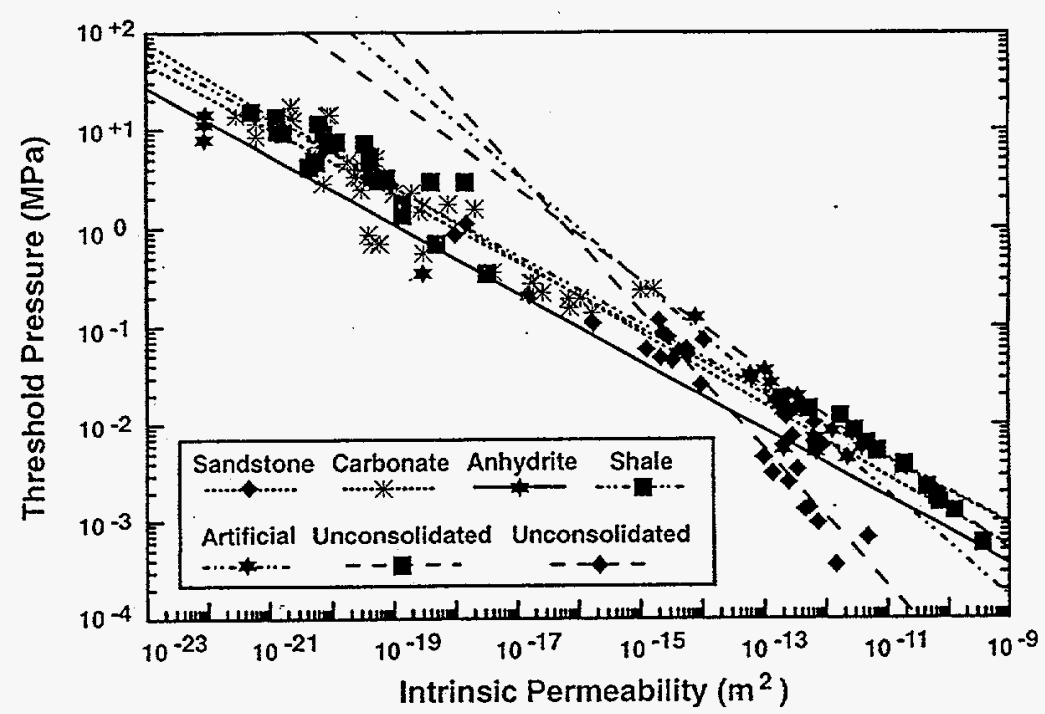

Figure 8. Literature data and correlations compiled by Davies (1991) between intrinsic permeability and threshold pressure.

Alone among two-phase properties, threshold pressure is amenable to measurement through field tests because it does not rely on determination of relative saturations. Provided that a medium is known to be fully brine saturated, its threshold pressure can be determined through a gas-injection test. Three in situ threshold-pressure tests were conducted in anhydrite interbeds above and below the WIPP repository in which permeability tests had already been completed. These three tests provided estimates of threshold pressure ranging from 0.27 to $0.01 \mathrm{MPa}$ for permeabilities ranging from $7 \times 10^{-20}$ to $3 \times 10^{-18} \mathrm{~m}^{2}$. The optimal testing procedure determined from these tests is as follows: 1) Isolate the interbed to be tested with packers and wait for the brine pressure to stabilize. 2) Inject gas (nitrogen) at the top of the test zone while withdrawing brine from the bottom, perturbing the pressure as little as possible. 3) After the brine in the test zone is completely replaced by gas, allow the test-zone pressure to stabilize. 4) Inject gas at a constant mass rate $\left(1\right.$ to $3 \mathrm{~cm}^{3} / \mathrm{min}$ at STP) while monitoring the pressure increase in the test zone and plotting pressure change and its derivative with respect to log time versus elapsed time on a log-log plot. Threshold pressure is reached when the pressure-change data and, more clearly, the pressure-derivative data deviate from the unit-slope line on the plot characteristic of wellbore storage (Figure 9 ). That is, until gas begins to penetrate the formation, the pressure in the test zone will rise linearly as gas is injected. Once gas begins to move out in the rock, the rate of pressure increase in the test zone will decrease.

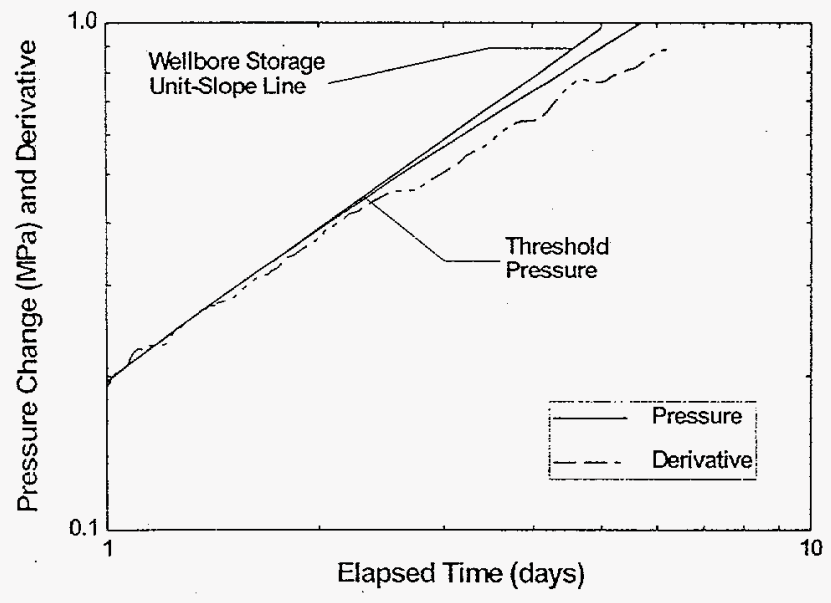

Figure 9. Determination of threshold pressure from pressure and pressure-derivative responses during gas injection. 
Additional information can be obtained by shutting in the test zone after the threshold pressure is surpassed and observing the subsequent pressure falloff as gas continues to flow from the test zone into the formation. The pressure decline should cease at the threshold pressure, providing a check on the estimate of threshold pressure obtained during the injection phase of the test. Operationally, the pressure-falloff phase can either be allowed to proceed at its own rate or it can be accelerated by venting small amounts of gas to decrease the pressure in steps and observing the pressure response after each step to determine the pressure at which gas stops flowing into the formation (Figure

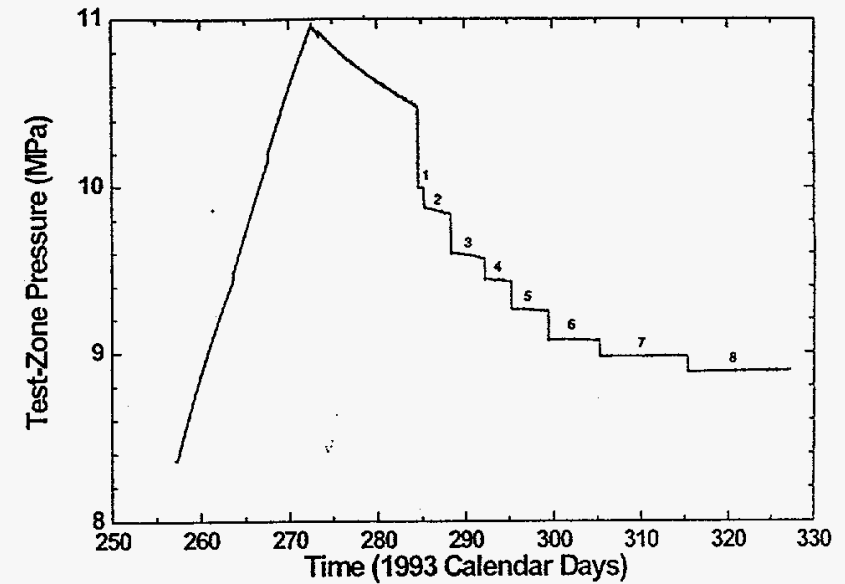

Figure 10. Example of stepwise pressure-falloff approach to determining threshold pressure. 10). Above the threshold pressure, the immediate response after each pressure drop will be an increase in the test-zone pressure as the local equilibrium between the test zone and the surrounding rock is restored, followed by a decrease in the test-zone pressure as outward flow resumes (Figure 11a). After a drop below the threshold pressure, the test-zone pressure will increase as gas flows back from the formation to the test zone (Figure 11b), ultimately to stabilize at the threshold pressure. Additional information might also be obtained by repeating the gas-injection test at different injection rates, but saturation uncertainties become more problematic as the test increases in complexity.

As shown on Figure 12, both the laboratory and field values of threshold pressure are lower than the correlation of Davies (1991) for anhydrite would predict. Several factors might contribute to this apparent discrepancy. First, the literature data (from Ibrahim et al., 1970) are sparse: only seven values are given, two of which are presented as upper bounds only and one as a lower bound only. Thus, the correlation is not well defined. Second, the literature data are from a salt dome caprock which may have a different sedimentological structure and, therefore, different flow properties than the bedded anhydrites at

a) Step 7 of 8
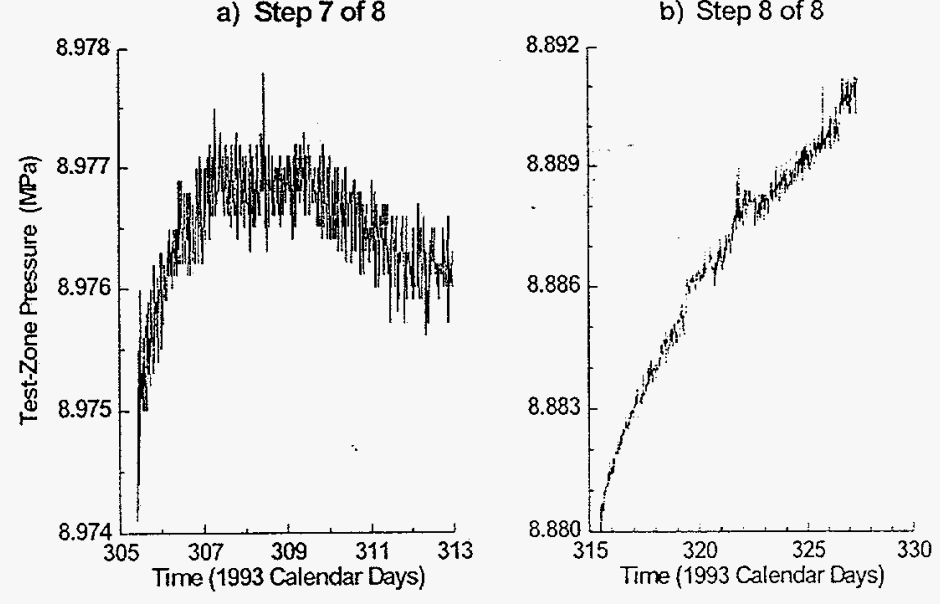

Figure 11. Example of bracketing threshold pressure between pressure-falloff steps (a) above threshold pressure and (b) below threshold pressure. WIPP. Third, the literature data may reflect the threshold pressure required to enter the anhydrite matrix whereas at WIPP we believe the gas is entering fractures. Fourth, the "permeabilities" used to plot the WIPP data on Figure 12 do not directly represent the fractures that gas is probably entering, but instead represent the average permeabilities of the entire thicknesses of anhydrite tested $(2.5 \mathrm{~cm}$ to $1 \mathrm{~m})$. The true fracture permeabilities must be higher than the thickness-averaged values, but exact values cannot be determined. 
Regardless of how well the WIPP data agree with the correlation of Davies, the laboratory and field experiments completed to date have shown that the threshold pressures of the anhydrite interbeds are low enough that gas may enter the interbeds at pressures well below the hydraulic-fracturing pressures. Thus, further field experimentation and model development to examine the effects of high threshold pressures in anhydrite interbeds are not warranted.

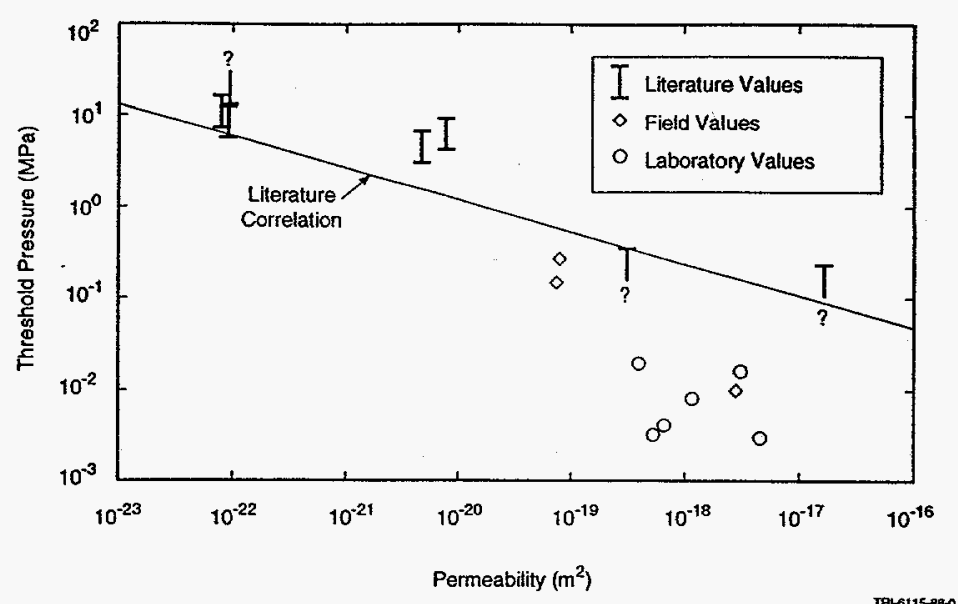

Figure 12. Comparison of literature and measured values for anhydrite threshold pressure.

\section{Pressure-Dependent Permeability}

Early modelers (e.g., Davies et al., 1992; Webb, 1992a) examining the effects of gas-generated pressures on repository performance assumed that the permeability and porosity of anhydrite interbeds would remain constant regardless of how pressures changed. They found that under some conditions, pressures in the repository could exceed lithostatic (Figure 13) because gas was generated more rapidly than it could flow away through the interbeds. Pressures several MPa greater than lithostatic were not considered realistic, however. Mendenhall et al. (1992) argued that either existing fractures in the interbeds would dilate or new fractures would be created as the pressure in the repository increased until the resulting enhanced permeability and porosity were adequate to accommodate the gas being generated. One potentially undesirable side effect of this enhanced

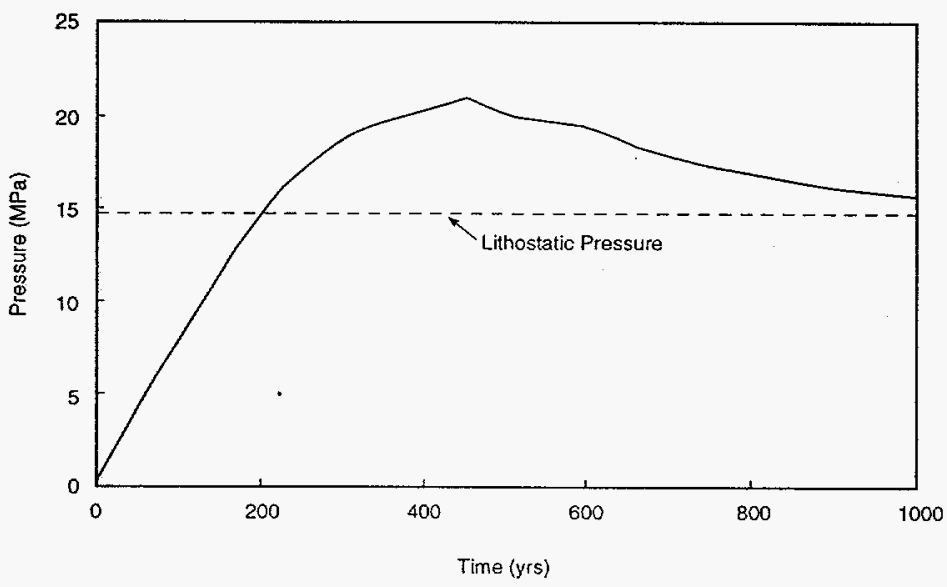
permeability was an increase in the distance to which gas would migrate from the repository.

Early scoping studies were conducted assuming interbed permeability increased above a defined "fracture" pressure while the porosity was assumed to remain constant. Results indicated that the repository pressure was easily capped by increasing the interbed permeability. However, the increase in gas-migration distance was large. Subsequently, WIPP Performance Assessment (PA) modelers began to consider more sophisticated ways of incorporating pressure-dependent interbed permeability and porosity in their models at the same time as field and laboratory experiments began 
to study the phenomenon. Laboratory tests on eight core samples showed that interbed permeabilities decreased by factors ranging from 1.6 to 5.3 as the net confining pressure was increased from 2 to 10 $\mathrm{MPa}$, with 52 to 92 percent of the reduction occurring as the confining pressure was increased from 2 to $6 \mathrm{MPa}$ (Figure 14). This behavior would translate to an increase in interbed permeability around the repository as pore pressures in the interbeds increased, thereby decreasing the effective confining pressure. One experiment was performed to examine the effect of pore pressure on permeability in the field. This experiment was run with individual constantpressure flow tests at four different pressures, one test at a pressure below the ambient pressure in the interbed and three tests at pressures above ambient. These tests showed a threefold increase in permeability as the test pressure was increased from about 7.5 to $10.8 \mathrm{MPa}$, with the rate of permeability increase accelerating as the pressure increased (Figure 15). Thus, both the laboratory and field measurements showed pressure-dependent permeability to be a plausible mechanism to relieve high gas pressures generated within the repository.

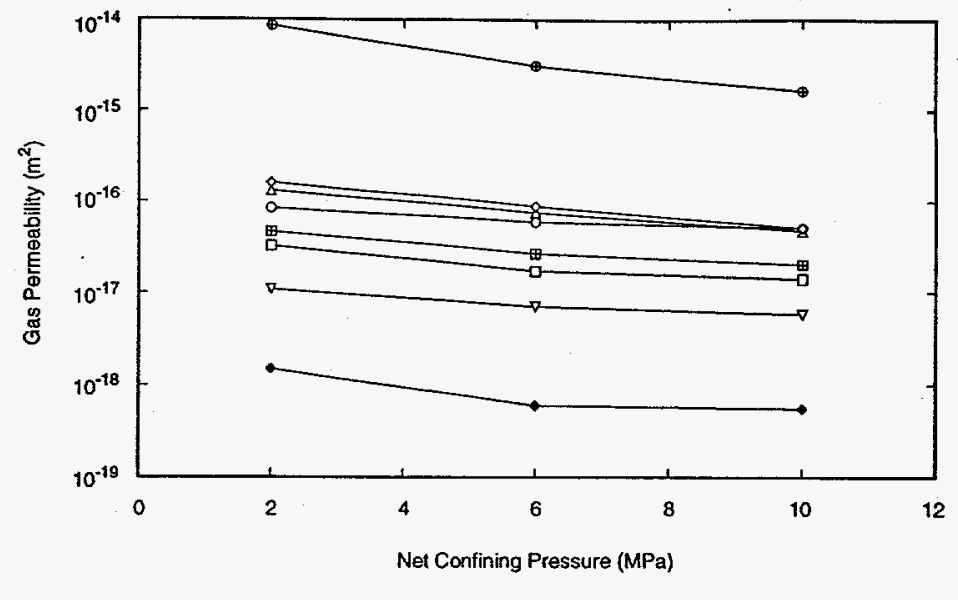

Figure 14. Laboratory data showing reduction in permeability as confining pressure increases.

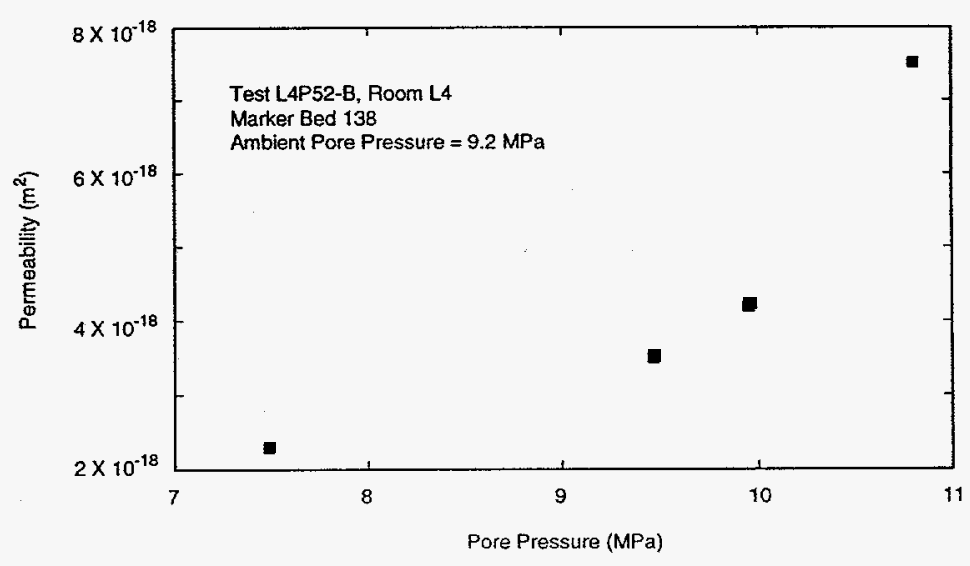

TR16175-92-0

Figure 15. Field data showing increase in permeability as induced pore pressure increases.

For PA purposes, a relatively simple, single-continuum model was developed that relates interbed porosity and permeability to pressure by first assuming a pressure-dependent compressibility. The pressure dependence is assumed to be initiated at a pore pressure slightly in excess of the estimated far-field pressure. Below this "initiating pressure $\left(\mathrm{P}_{\mathrm{i}}\right)$," anhydrite compressibility and permeability are treated as constant. For pressures above the initiating pressure, compressibility is assumed to increase linearly with pressure up to a so-called "fully altered pressure $\left(\mathrm{P}_{\mathrm{a}}\right)$," above which no further property alteration occurs. PA related porosity $(\phi)$ to pressure $(P)$ through a definition of compressibility (C) given by: 


$$
C=\frac{1}{\phi} \frac{d \phi}{d P}
$$

Porosity as a function of pore pressure is obtained by integration of the compressibility equation. This results in the porosity function shown in Figure 16 where the ratio of porosity to intact porosity is a function of pore pressure. By analogy to the parallel plate approach to determining fracture permeability, in which permeability is proportional to a power of fracture aperture (or porosity), altered permeability is related to altered porosity by:

$$
\frac{k}{k_{i}}=\left[\frac{\phi}{\phi_{i}}\right]^{J}
$$

where: $k=$ permeability of altered anhydrite,

$$
\begin{array}{lll}
k_{i} & = & \text { permeability of intact anhydrite, } \\
\Phi & = & \text { porosity of altered anhydrite, } \\
\phi_{i} & = & \text { porosity of intact anhydrite, and } \\
J & = & \text { an empirical parameter. }
\end{array}
$$

This model relies on the specification of "fully altered" values of porosity $\left(\phi_{\vec{a}}\right)$ and permeability $\left(k_{a}\right)$. PA handles uncertainty in these values by sampling each of them from ranges determined to be reasonable based on available geologic and hydrologic information. The empirical parameter $\mathrm{J}$ given above takes whatever value is necessary to provide the sampled value of fully altered permeability given the sampled value of fully altered porosity. Figure 16 shows conceptually how permeability increases much more rapidly than porosity as the pore pressure increases.

Preliminary calculations using this model showed that it was effective at relieving gas pressure in the repository; the fully altered pressure was not reached for any sampled combination of parameters. However, the calculated gas-migration distance was found to be highly sensitive to the sampled values. High porosity ranges coupled with low permeability ranges led to low migration distances and low porosity ranges coupled with high permeability ranges led to high migration distances. Without data to narrow the sampled ranges, gas-migration distances would remain highly uncertain.

Because the exact form of the relationship between pore pressure and permeability is unknown, a second model relating the two was developed. In contrast to the socalled "porosity" model developed by PA, the second model, termed the "aperture" model, assumes that increasing pore pressures cause

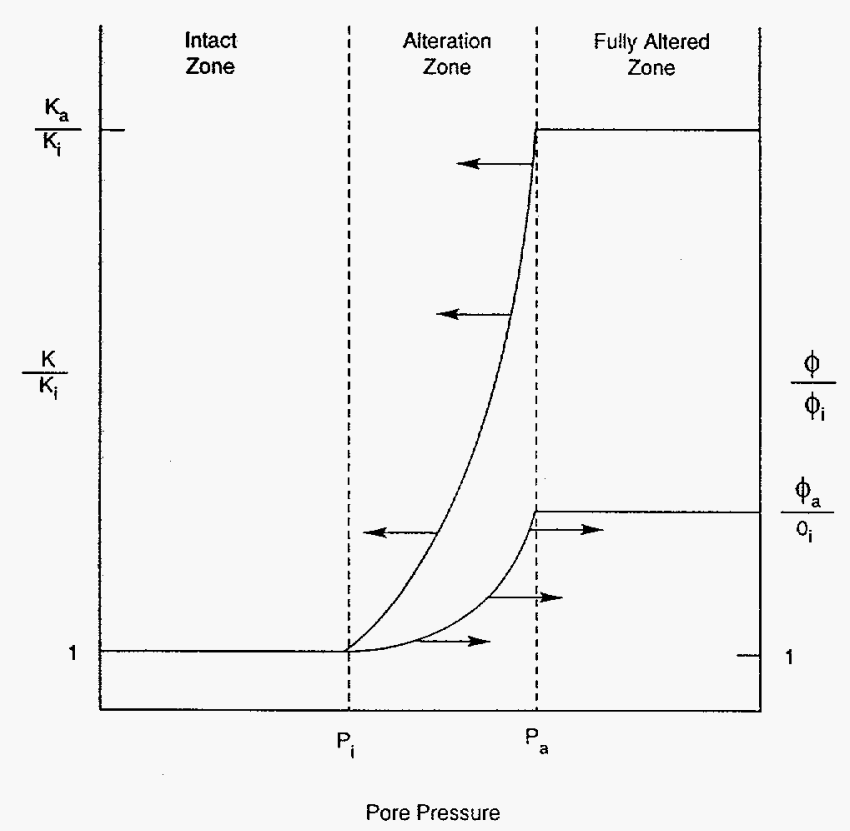

Figure 16. PA "porosity" model for pressure-dependent porosity and permeability. 
fracture apertures to increase and calculates the permeability of the dilated fractures using the cubic law. The overall porosity (aperture) change is related to pressure change through Eq. 1 above and then permeability is calculated from:

$$
k=\frac{n b^{3}}{12 h}+k_{m}
$$

where: $n \quad=\quad$ number of fractures among which the porosity is distributed,

$\begin{array}{lll}b & = & \text { average fracture aperture, } \\ h & = & \text { thickness of rock matrix, } \\ k_{m} & = & \text { matrix permeability. }\end{array}$

A drawback associated with this equation is that the parameter $n$ (number of fractures) must be either known or estimated.

For a given porosity change, the porosity and aperture models predict greatly different permeability changes. Figure 17 shows how calculated permeabilities differ between the two models for a one percent porosity change, assuming an initial (unaltered) permeability of $10^{-19} \mathrm{~m}^{2}$ and ranges of values of the parameters $J$ and $n$ in Eq. 2 and 3 , respectively. The initial condition used for the aperture-model calculations shown in Figure 17 may be somewhat unrealistic because, by starting with an overall permeability equal to the assumed matrix permeability, the model effectively assumes that no fractures exist initially. Regardless of the reasonableness of this starting assumption, however, the aperture model shows that permeabilities calculated using a cubic-law expression such as Eq. 3 are several orders of magnitude higher than those predicted by the porosity model for a given porosity (total fracture aperture).

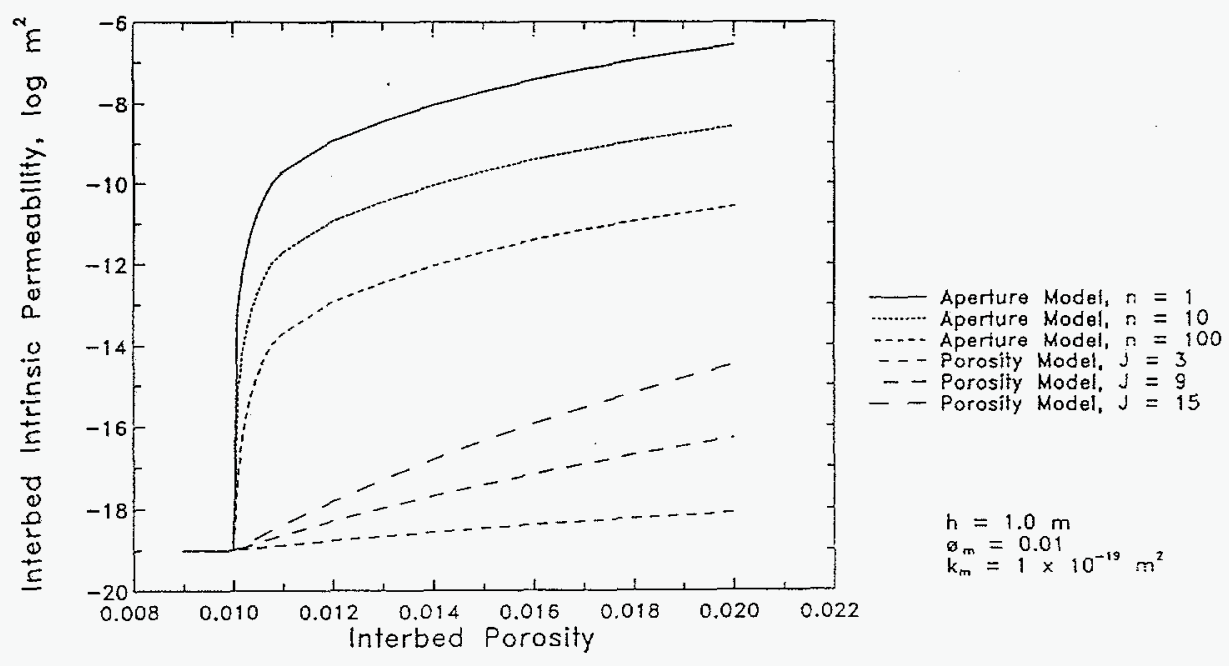

Figure 17. Comparison of permeabilities calculated by porosity and aperture models for a one percent porosity change.

Use of the porosity and aperture models to predict gas-migration distances also results in significantly different consequences. Figure 18 shows how the predicted porosity changes directly above a waste-storage room over a period of 10,000 years differ between the two models. The porosity model predicts much greater increases in porosity, particularly within the first $2000 \mathrm{yr}$, than the aperture model. Increasing the porosity has the effect of decreasing the gas-migration distance, as 
shown in Figure 19. To access the storage volume needed for gas pressure to reach equilibrium with the far-field brine pressure, gas must travel a much greater distance under aperture-model assumptions than under porosity-model assumptions.

These calculations have shown the importance of understanding the functional relationships among pressure, porosity, and permeability changes in the anhydrite interbeds if accurate calculations of gas-migrations distances are to be performed. An effort was made to fit the porosity and aperture models to the field data already collected relating permeability to pore pressure (Figure 15) to determine if either or both was inconsistent with those data. By modifying values of parameters such as initiating pressure, both models could be made to fit the data, as shown in Figure 20.

To distinguish between the two models (or develop a different, more appropriate model), not just permeability change but also porosity change needs to be measured as a function of pressure. Consequently, a new test tool has been developed to measure the dilation of the overall thickness of anhydrite interbeds during permeability testing over a range of pressures. The tool is conceptually similar to the Pac-ex system developed at the Canadian Underground Research Laboratory (Thompson et al., 1989), but is adapted to a corrosive high-pressure environment. The tool uses linear variable-differential transformers (LVDT's) to measure changes in the separation of anchors set above and below interbeds during testing (Figure 21). This tool will provide information only on the total amount of dilation that occurs, not on the number of fractures dilating. Nevertheless, the data can be used to determine the appropriateness of the porosity model

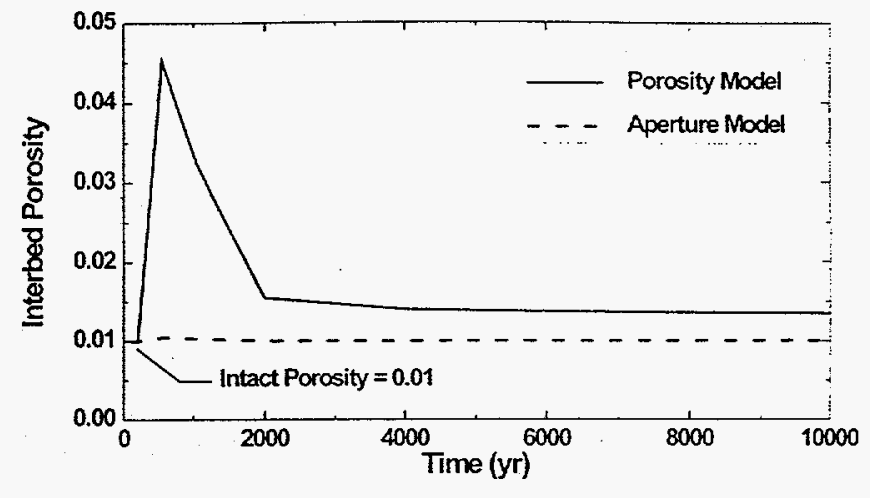

Figure 18. Comparison of interbed porosities above disposal room calculated using porosity and aperture models.

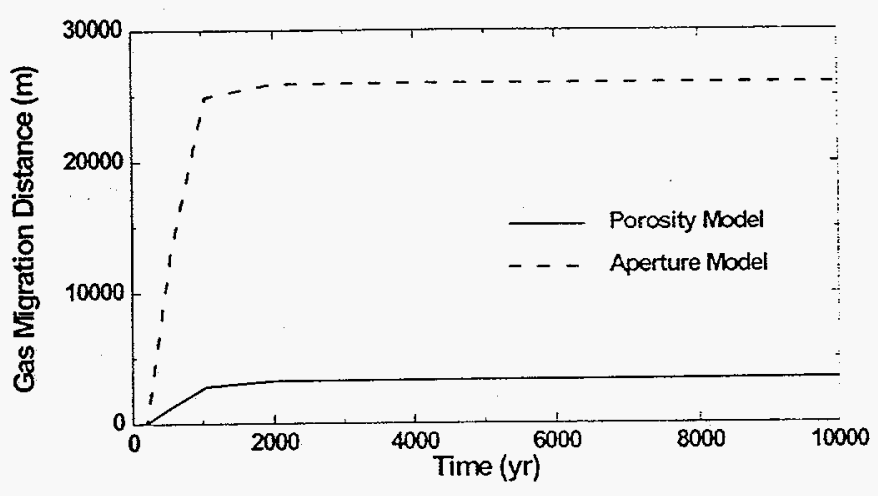

Figure 19. Comparison of gas-migration distances calculated using porosity and aperture models.

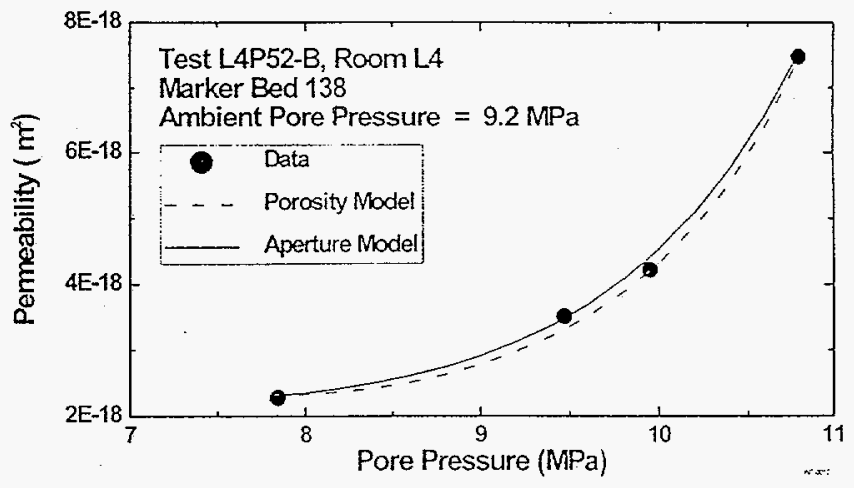

Figure 20. Example matches of porosity and aperture models to available field data. 
and the aperture model (assuming a "reasonable" range for the number of affected fractures).

\section{Summary and Conclusions}

The initial models used by WIPP PA to model the gas generation and migration scenario were simplified because of a lack of WIPP data on important processes and parameters. Two sets of two-phase characteristic curves taken from studies reported in the literature were used in PA and other models to evaluate their effects on gas release. Calculated gas-migration distances were found to be strongly dependent on the nature of the characteristic curves used, motivating laboratory experiments on core samples to define WIPP-specific characteristic curves for anhydrite. The information obtained from the laboratory program may be used directly in future simulations of gas migration, with appropriate upscaling. A correlation between anhydrite permeability and threshold pressure derived from literature data, combined with in situ permeability data, suggested that

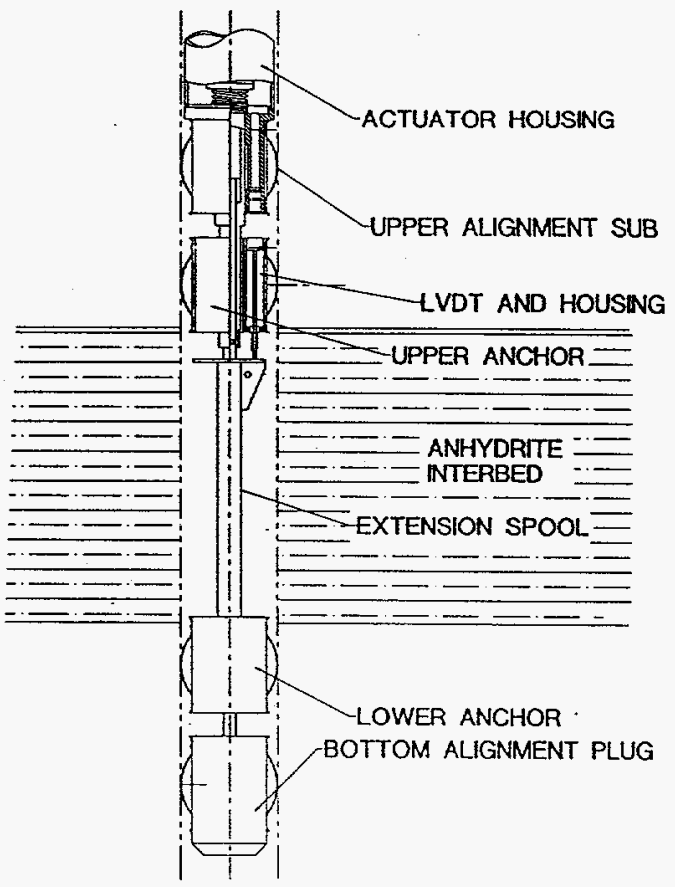

Figure 21. Schematic of tool to measure interbed dilation. gas might not be able to enter anhydrite interbeds around the WIPP repository at pressures below the hydraulic fracturing pressure. Both laboratory and field experiments have shown that threshold pressures in the interbeds are much lower than predicted by the literature correlation, and reaching the hydraulic fracturing pressure before gas entry is no longer of concern. Early models treating fracture apertures and permeabilities as constant and independent of effective stress allowed repository pressures to reach unrealistically high levels above lithostatic pressure because gas could not leave the repository as fast as it was generated. Field and laboratory experiments showed that anhydrite fractures would dilate and increase in permeability as pore pressures increased, but did not provide enough information to define the functional relationship between pressure and fracture aperture and permeability. Two models relating pressure to permeability and aperture in different ways were developed and were shown to predict significantly different gas-migration distances. A field experimental program has been developed to provide direct measurements of permeability and aperture change as functions of pressure to allow development of a realistic model coupling rock mechanics with fluid flow.

These examples illustrate the WIPP project approach of performing iterative modeling and experimental activities to, first, determine the importance of different processes and parameters and, second, reduce uncertainty in those areas that have the largest effect on the calculated performance of the repository. This approach reduces expenditures in areas that have little impact on regulatory compliance and provides assurance that the most important parts of the system receive adequate attention.

\section{References}

Beauheim, R.L., R.M. Roberts, T.F. Dale, M.D. Fort, and W.A. Stensrud. 1993. Hydraulic Testing of Salado Formation Evaporites at the Waste Isolation Pilot Plant Site: Second Interpretive Report. SAND92-0533. Albuquerque, NM: Sandia National Laboratories. 
Beauheim, R.L., G.J. Saulnier, Jr., and J.D. Avis. 1991. Interpretation of Brine-Permeability Tests of the Salado Formation at the Waste Isolation Pilot Plant Site: First Interim Report. SAND90-0083. Albuquerque, NM: Sandia National Laboratories.

Brooks, R.H., and A.T. Corey. 1964. Hydraulic Properties of Porous Media. Hydrology Paper No. 3. Ft. Collins, CO: Colorado State University.

Davies, P.B. 1991. Evaluation of the Role of Threshold Pressure in Controlling Flow of WasteGenerated Gas into Bedded Salt at the Waste Isolation Pilot Plant. SAND90-3246. Albuquerque, NM: Sandia National Laboratories.

Davies, P.B., L.H. Brush, and F.T. Mendenhall. 1992. "Assessing the Impact of Waste-Generated Gas from the Degradation of Transuranic Waste at the Waste Isolation Pilot Plant (WIPP): An Overview of Strongly Coupled Chemical, Hydrologic, and Structural Processes," Proceedings: Workshop on Gas Generation and Release from Radioactive Waste Repositories, Aix-en-Provence, France, September 23-26, 1991. Paris, France: OECD Nuclear Energy Agency. 54-74.

Ibrahim, M.A., M.R. Tek, and D.L. Katz. 1970. Threshold Pressure in Gas Storage. Project 26-47 of the Pipeline Research Committee, American Gas Association at The University of Michigan. Arlington, VA: American Gas Association, Inc.

Mendenhall, F.T., B.M. Butcher, and P.B. Davies. 1992. "Investigations Into the Coupled Fluid Flow and Mechanical Creep Closure Behavior of Waste Disposal Rooms in Bedded Salt," Proceedings: Workshop on Gas Generation and Release from Radioactive Waste Repositories, Aix-en-Provence, France, September 23-26, 1991. Paris, France: OECD Nuclear Energy Agency. 282-295.

Parker, J.C., R.J. Lenhard, and T. Kuppusamy. 1987. "A Parametric Model for Constitutive Properties Governing Multiphase Flow in Porous Media," Water Resources Research. Vol. 23, no. 4, 618-624.

Preuss, K. 1987. TOUGHUser's Guide. NUREG/CR-4645, SAND86-7104, LBL-20700. Washington, DC: US Nuclear Regulatory Commission.

Thompson, P.M., E.T. Kozak, and C.D. Martin. 1989. "Rock Displacement Instrumentation and Coupled Hydraulic Pressure/Rock Displacement Instrumentation for Use in Stiff Crystalline Rock," Proceedings: Workshop on Excavation Response in Geological Repositories for Radioactive Waste, Winnipeg, Canada, April 26-28, 1988. Paris, France: OECD Nuclear Energy Agency. 257-270.

Van Genuchten, M.T. 1980. "A Closed-Form Equation for Predicting the Hydraulic Conductivity of Unsaturated Soils," Soil Science Society of America Journal. Vol. 44, 892-898.

Webb, S.W. 1992a. "Sensitivity Studies for Gas Release from the Waste Isolation Pilot Plant (WIPP)," Proceedings: Workshop on Gas Generation and Release from Radioactive Waste Repositories, Aix-en-Provence, France, September 23-26, 1991. Paris, France: OECD Nuclear Energy Agency. 309-326.

Webb, S.W. 1992b. "Steady-State Saturation Profiles for Linear Immiscible Fluid Displacement in Porous Media," Heat and Mass Transfer in Porous Media, ASME HTD, Vol. 216, 35-46.

WIPP Performance Assessment Department. 1993. Preliminary Performance Assessment for the Waste Isolation Pilot Plant, December 1992, Volume 4: Uncertainty and Sensitivity Analyses for 40 CFR 191, Subpart B. SAND92-0700/4. Albuquerque, NM: Sandia National Laboratories. 